\title{
Opinión expresada sobre un programa de formación de formadores para profesionales que trabajan con UDls
}

\author{
InSÚA, P. ${ }^{(1)}$; MONCADA, S. ${ }^{(2)}$ \\ (1) Universidad del País Vasco (UPV/EHU). \\ (2) Delegación del Gobierno para el Plan Nacional sobre Drogas.
}

Enviar correspondencia a:

Dra. Patricia Insúa. Facultad de Psicología, Av. de Tolosa, 70, 20018 San Sebastián. Teléfono 943 015735. E-mail: pbpincep@sc.ehu.es.

\section{RESUMEN:}

Objetivo: La necesidad de poner en marcha y evaluar Programas de prevención de los riesgos asociados al uso de drogas con los usuarios de éstas es, hoy por hoy, incuestionable por numerosas razones.

Presentamos la evaluación de proceso de un Programa de Formación de Formadores diseñado en una estrategia de redes, puesto en marcha con profesionales sanitarios de todo el Estado, con el objetivo de capacitarles para diseñar, implementar y evaluar este tipo de Programas de prevención con la población de usuarios de drogas.

Material y Método: Se estudia la opinión expresada por los 56 profesionales sanitarios sobre los materiales de apoyo diseñados y los aspectos estructurales del Programa de Formación al que han asistido a través de un cuestionario diseñado al efecto.

Resultados: Encontramos una opinión muy positiva de todas las áreas evaluadas tanto en lo que respecta a la satisfacción general e implicación de los participantes en el Programa como a la utilidad e interés de los contenidos trabajados y a la adecuación de la metodología aportada.

Conclusión: Esta evaluación ha permitido establecer el control de calidad de este Programa para Formadores y mostrar objetivamente que es adecuado para la población para la que fue diseñado. Su estructuración como una estrategia de redes ha permitido que estos 56 profesionales formen a 684 profesionales más de sus propias Comunidades Autónomas, demostrando que una estrategia de redes bien organizada permite optimizar los recursos disponibles y posibilitando que estos profesionales diseñen y pongan en marcha este tipo de Programas con los usuarios de drogas.

Palabras clave: Evaluación. Programas de Prevención. Sida. Drogodependencias. Formadores.

\begin{abstract}
:
Objective: The need to implement AIDS Prevention Programmes with drugs users is unquestionable for many reasons. We present the process evaluation of a Training Programme designed like a Network Programme carried out with sanitary professionals in our country. The aim of this Programme is to train these professionals to design, implement and evaluate Prevention Programmes of health risks associated to drug use for their clients.

Method: We study the expressed opinion of 56 sanitary professionals after they have received the Programme about the support materials designed for it and its structural aspects.

Results: We found a very positive opinion about all the evaluated areas. Both of the general satisfaction and personal implication in the workshop and of the usefulness and interest of the contents and the fitness of the methodology.

Conclusions: This evaluation had permitted to establish the quality control of this Training Programme and to know objectively that is suitable for the population for which it was designed. This Programme was designed to organise a network strategy and was replicated for these professionals in the different provinces where they live. From 56 professionals trained in the first stage after one year there are in our country 684 professionals trained to carry out this kind of Programmes with drug users.
\end{abstract}

Key words: Evaluation. Programmes. Aids Prevention. Drugs use. Trainning. 


\section{INTRODUCCIÓN}

$\mathbf{L}$ a necesidad insoslayable de trabajar con los individuos usuarios de drogas en programas de prevención de problemas asociados al uso de drogas, es señalada por distintos autores (Bayés, 1995; Des Jairlais et al, 1999; Freudenberg, 1990; Mantell, DiVittis y Auerbach, 1997) y ya la hemos desarrollado en otro trabajo (Insúa y Moncada, en prensa).

En el caso de España el Sida y la infección por VIH se han convertido en el principal problema de salud asociado al uso de drogas. Una característica peculiar de la epidemia en nuestro país es que el $65 \%$ de los enfermos son o han sido usuarios de drogas por vía intravenosa y a pesar del descenso de la conducta de inyección de drogas, aún existe un importante porcentaje de usuarios que utilizan esta vía constatándose prevalencias muy elevadas de prácticas de riesgo en este colectivo. Además, un porcentaje importante de los inyectores de drogas tienen parejas que no se inyectan ellas mismas y que sin embargo, se encuentran en riesgo de transmisión de distintas patologías a través de la vía sexual.

En los últimos años se ha realizado un considerable esfuerzo desde distintos ámbitos de atención a los problemas derivados del consumo de drogas para que la prevención de la transmisión del VIH esté presente como uno de los objetivos prioritarios. A pesar del incremento de los programas de mantenimiento con sustitutivos y, en general, de la apertura de los dispositivos terapéuticos de atención a drogodependientes hacia objetivos más realistas de mejora de la calidad de vida, es necesario fomentar iniciativas para promover la educación para la salud entre los usuarios de drogas que acuden a tratamiento.

Pero los profesionales que trabajan en los centros de atención a drogodependientes (médicos generales, psiquiatras, psicólogos, profesionales de enfermería, trabajadores sociales y otros) aunque conocen la necesidad de trabajar con sus clientes la modificación positiva de los hábitos de riesgo, precisan una formación adecuada para aprender a transmitir este tipo de mensajes y para hacer educación para la salud. Por ello resulta necesario proporcionarles las herramientas de trabajo necesarias para que puedan modificar los comportamientos de riesgo para la transmisión de los riesgos asociados al uso de drogas.

Con esta finalidad, en 1998, los Ministerios de Sanidad y Consumo e Interior, firmaron un Protocolo de Intenciones de Colaboración para la puesta en marcha de Programas de prevención de la transmisión del $\mathrm{VIH} /$ Sida entre usuarios de drogas, a través de la Secretaria de Plan Nacional sobre el Sida y la Delegación del Gobierno para el Plan Nacional sobre Drogas (DGPNSD). Este acuerdo tiene como objetivo promo- ver intervenciones preventivas en las redes de asistencia al drogodependiente de las 17 Comunidades Autónomas y las Ciudades de Ceuta, Melilla, Madrid y Barcelona.

El proyecto contempla un plan de formación en redes para la implementación de Talleres para la modificación de las prácticas de riesgo tanto de la conducta sexual como de la de consumo, en la forma de Talleres de Sexo más Seguro y Talleres de Consumo de menor Riesgo dirigido a los profesionales de los centros de tratamiento de todos los Planes de Drogas, tanto Autonómicos como de las Ciudades de Ceuta y Melilla, Madrid y Barcelona.

De forma sintética, este Proyecto contempla una primera fase de Formación de Formadores (al menos dos por cada territorio) y una segunda en la que estos formadores realizan cursos en cada CC.AA. dirigidos a los profesionales que trabajan en los Centros de atención a drogodependientes con el objetivo último de que en un período de dos años desde el inicio del programa los profesionales que trabajan en los centros de atención al drogodependiente puedan realizar este tipo de Talleres con usuarios de drogas.

Para la realización del proyecto la Delegación del Gobierno para el Plan Nacional sobre Drogas firmó un Convenio de Colaboración con la Universidad del País Vasco, a través de su Departamento de Psicología Básica. La colaboración entre las dos instituciones aporta importantes beneficios para ambas partes ya que la Universidad aporta los aspectos teóricos y metodológicos, y las redes de profesionales que participaron en el programa permiten el ajuste del mismo a las condiciones y características de su práctica profesional, consiguiéndose de esta manera una interesante conexión entre la teoría y la práctica (entre la investigación y la acción) que redunda en beneficio del Programa global.

\subsection{Evaluación de la primera fase del Programa de Formación de Formadores para la prevención del VIH/Sida entre usuarios de drogas.}

A la hora de diseñar un Programa de intervención con un determinado colectivo, tenemos que tener en cuenta los objetivos de dicho Programa y, en función de esos objetivos, establecer el tipo de contenidos que se van a trabajar en él. De la misma manera, una vez establecidos los contenidos teóricos, tenemos que diseñar otros aspectos estructurales de dicho Programa (contexto situacional, duración, metodología de trabajo, estilo de los docentes, etc.) que van a ser también fundamentales para su puesta en marcha. Una vez diseñado el Programa, tenemos que organizar el diseño de la evaluación que tiene que implicar tanto aspectos de impacto y resultados del programa 
(vgr. la evaluación sumativa), como aspectos formativos y de implementación (vgr. la evaluación de proceso) (Mantell et al., 1997).

La evaluación sumativa valora la cuestión de la efectividad de una intervención determinada. Esta cuestión se valora en dos niveles: evaluación de impacto y evaluación de resultados. Si bien estos términos (impacto y resultados) son utilizados de distintas maneras por los diversos autores, podríamos considerar que la "evaluación de impacto" analiza el grado en el cual la intervención preventiva ha obtenido sus objetivos (se valora el impacto inmediato de la intervención) y que la "evaluación de resultados" tiene un foco más amplio, basado en la comunidad, en el cual se valoran las metas logradas a largo plazo (se valora el resultado global de la intervención sobre la comunidad). Ambos métodos de evaluación sumativa incluyen estrategias de recogida de datos cuantitativas y cualitativas.

Por su parte, la evaluación de proceso, implica una variedad de técnicas que permiten al evaluador valorar la calidad de un Programa de prevención. La evaluación formativa se focaliza en la valoración de las necesidades de la comunidad y en determinar la idoneidad de los instrumentos de evaluación, mientras que la evaluación de implementación valora cómo de bien el plan de intervención original fue implementado y la opinión de los participantes sobre el mismo. Diversos métodos de valoración son usados en la evaluación de proceso y se también se usan técnicas cualitativas y cuantitativas para recoger la información.

El presente artículo expone la opinión expresada sobre el Programa de Formación de Formadores por los 56 profesionales de las redes de atención de las distintas CC.AA formados en la segunda fase del mismo.

\section{MATERIALY MÉTODOS}

El Programa de Formación de Formadores que hemos diseñado para poner en marcha con profesionales de la Red Pública que trabajan con el colectivo de usuarios de drogas, tiene sus fuentes teóricas en los Modelos sociocognitivos del cambio de comportamiento (Insúa, 1999a), y se consolida después de haber sido puesto a prueba y evaluado en distintas poblaciones de profesionales sanitarios del Estado durante los últimos años (Insúa, 1999b). Las evaluaciones de cada uno de los Programas previamente realizados anteriormente han permitido estructurar un Programa de intervención en Redes que consta de diferentes fases:
1) La primera fase, fue el contacto con los responsables de los Planes Autonómicos de Drogodependencias de las distintas CC.AA. y de las ciudades de Ceuta y Melilla, así como de 4 ONG de carácter supracomunitario como son Cruz Roja, Cáritas, Proyecto Hombre y UNAD; quienes seleccionaron a los profesionales que iban a ser formados y a continuar el trabajo en las CC.AA. En esta fase también se desarrolló el diseño de los Talleres específicos que iban a componer los Cursos de Formación.

2) La segunda fase se concretó en la formación de los profesionales seleccionados quienes, además, supervisaron el borrador de un Manual de Educación Sanitaria redactado para este Programa, aportando su visión de los distintos capítulos, que fue incorporada en la edición final del mismo que se entregó como material de apoyo teórico.

3) La tercera fase, supuso el diseño y realización ${ }^{2}$ de Programas de Prevención de la Transmisión del VIH por parte de estos profesionales formados, con profesionales sanitarios de las redes públicas de asistencia a las drogodependencias de sus respectivas CC.AA.

4) La cuarta fase supone la realización de estos Programas de Prevención con distintos grupos de usuarios de drogas del Estado.

En este Programa, la evaluación formativa fue llevada a cabo por la Delegación del Gobierno para el Plan Nacional sobre Drogas y el Depto. de Psicología Básica de la UPV/EHU, valorando las necesidades en materia de atención a los usuarios de drogas en las distintas Comunidades Autónomas, y teniendo en cuenta las peticiones de formación de los profesionales sanitarios para afrontar Programas de reducción de los daños asociados al uso de drogas entre sus clientes ( $1^{\text {a }}$ fase)

Teniendo en cuenta esta evaluación se diseñó un Programa para la Formación de los profesionales seleccionados por las distintas CC.AA. ( $2^{\text {a }}$ fase) que consta de 32 horas de duración con dos Cursos de 16 horas cada uno:

a) Programas de Prevención de la Transmisión Sexual del VIH/SIDA, en la forma de "Talleres de Sexo Más Seguro" (TSMS) (16 horas).

b) Programas de Prevención de la Transmisión Parenteral del VIH/SIDA, en la forma "Talleres de Consumo de Menos Riesgo" (TCMR) (16 horas).

Ambos cursos fueron implementados en una semana, en régimen de internado, trabajando mañana

\footnotetext{
2 Se supervisó el diseño de todos los Talleres realizados, de tal manera que cumplieran los criterios científicos y metodológicos necesarios para conseguir los objetivos de formación que pretendían y homologar los conocimientos a transmitir.
} 
y tarde y de lunes a viernes. Se realizaron en total dos semanas de formación.

Los objetivos principales de dichos Cursos han sido:

-Incrementar los conocimientos y las habilidades de los profesionales formados de cara a que puedan fomentar la modificación de las prácticas de riesgo para la transmisión sexual y parenteral del VIH entre los sujetos usuarios de drogas.

-Ofrecer a estos profesionales los recursos teóricos y técnicos necesarios para la realización de Programas de Formación en la forma de "Talleres de Sexo Más Seguro" y "Talleres de Consumo de Menos Riesgo" con otros profesionales sanitarios de sus CC.AA. y de esta forma posibilitar el trabajo en redes:

a) Formar a formadores

b) Coordinar y supervisar el período de formación

c) Intercambiar conocimientos y experiencias

-Ofrecer los conocimientos necesarios que les permitan aprender y transmitir la metodología de evaluación correcta de estos Programas de Prevención, de tal manera que se puedan mejorar y objetivar proceso e impacto de los mismos.

Como materiales de apoyo teórico y metodológico a dicho Programa se han realizado ${ }^{3}$.

a) Un "Manual de Educación Sanitaria: Recursos para Diseñar Talleres de Prevención con Usuarios de Drogas" (Insúa, 1999a).

b) Dos vídeos con sus respectivas Guías de utilización. Ambos vídeos: "Hacia un sexo más seguro" (Insúa, 1999c) y "Si te metes, no te mates" (De Andrés, 1999), fueron elaborados como recursos psicopedagógicos para ayudar en la puesta en marcha de los Talleres.

Realizado un muestreo de cuotas desde el Plan Nacional sobre Drogas, se formaron 4 grupos de profesionales sanitarios ( $N=56)$, seleccionados por conveniencia ${ }^{4}$ por los responsables de los Planes de Drogas de las distintas CC.AA.; asistiendo a los cursos dos grupos la primera semana, y dos grupos la segunda semana. Estos grupos tenían aproximadamente el mismo número de profesionales.

Así, este Programa se concibe y se ofrece como un "paquete"; que incluye formación específica teórica y metodológica tanto sobre conducta sexual como sobre conducta de consumo de drogas; y materiales de apoyo (teóricos y metodológicos), que facilitan el diseño, la realización y la evaluación de nuevos Programas de prevención.
Además, estos Talleres han servido como modelo para que los profesionales formados diseñen sus propios Programas de intervención. Nuestra idea era que al trabajar en redes, la formación de un número limitado de profesionales sanitarios en el diseño, implementación y evaluación de este tipo de Programas de Prevención iba a servir para formar en un año a un número mucho mayor de profesionales sanitarios de las distintas CC.AA.

Nuestra intención en este artículo es profundizar en la evaluación de implementación del mismo, a través de los datos obtenidos con las respuestas de los 56 profesionales formados en la segunda fase del Programa.

Para la evaluación de implementación, a cada participante se le administró un cuestionario que recogió información sobre distintos aspectos subjetivos y estructurales de los Talleres, como son: valoración global, grado de satisfacción, utilidad, duración, participación, implicación, interés, información (suficiencia, contenidos), metodología (adecuación, dinámicas), materiales de apoyo aportados (manual y vídeo), docentes (actuación, claridad, credibilidad y empatía), y percepción de habilidades aportadas por el Curso para la realización de "Talleres de Sexo Más Seguro" y "Talleres de Consumo de Menos Riesgo".

Todas las preguntas han sido respondidas a través de escalas tipo Likert de 6 puntos, donde el 1 es la puntuación más negativa y el 6 la más positiva, a excepción del tiempo destinado a cada uno de los Cursos (ver Tabla 1).

Como se puede observar en la Tabla 1, las distintas preguntas se han distribuido en aspectos estructurales más genéricos de los Cursos de Formación a los que hemos denominado: Aspectos generales, Actitud del grupo, Información, Metodología, Materiales de Apoyo, Actuación de los docentes y Aportaciones de recursos teóricos y técnicos para diseñar Talleres.

Los cuestionarios han sido administrados de forma voluntaria y anónima tras finalizar los Cursos de Formación en todos los grupos. Los profesionales sanitarios formados han empleado aproximadamente una media de 15 minutos en autocumplimentar cada uno de ellos.

Todos estos indicadores nos ha permitido evaluar el grado de satisfacción de los profesionales con el Programa de Formación de Formadores, tanto con el Taller de Sexo más Seguro, como con el Taller de Consumo de menor Riesgo, permitiéndonos un "control de calidad" del mismo.

\footnotetext{
${ }^{3}$ Dichos materiales de apoyo se han distribuido a todos los profesionales formados en este Programa de Redes, a través de la Delegación del Gobierno para el Plan Nacional sobre Drogas.

${ }^{4}$ Las muestras de conveniencia se organizan en función de la oportunidad de los participantes (Mantell, DiVittis y Auerbach, 1997).
} 
TABLA 1: EVALUACIÓN DE PROCESO: CUESTIONARIO CURSO DE FORMACIÓN DE FORMADORES ENTSMSY ENTCMR.

\begin{tabular}{|c|c|c|}
\hline ASPECTO & VARIABLE & RANGO DE RESPUESTA \\
\hline $\begin{array}{l}\text { ASPECTOS } \\
\text { GLOBALES }\end{array}$ & $\begin{array}{l}\text { Valoración General } \\
\text { Satisfacción } \\
\text { Utilidad } \\
\text { Duración }\end{array}$ & $\begin{array}{l}1=\text { Totalmente Negativo a } 6=\text { Totalmente Positivo } \\
1=\text { Totalmente Insatisfecho a } 6=\text { Totalmente Satisfecho } \\
1=\text { Totalmente Inútil a } 6=\text { Totalmente Útil } \\
1=\text { Totalmente Corto a } 7=\text { Totalmente Largo }\end{array}$ \\
\hline ACTITUD GRUPO & $\begin{array}{l}\text { Participación } \\
\text { Implicación } \\
\text { Interés }\end{array}$ & $\begin{array}{l}1=\text { Nada Participativo a } 6=\text { Totalmente Participativo } \\
1=\text { Nada Implicado a } 6=\text { Totalmente Implicado } \\
1=\text { Totalmente Desinteresado a } 6=\text { Totalmente Interesa. }\end{array}$ \\
\hline INFORMACIÓN & $\begin{array}{l}\text { Suficiencia } \\
\text { Comprensibilidad } \\
\text { Credibilidad } \\
\text { Utilidad } \\
\text { Contenidos }\end{array}$ & $\begin{array}{l}1=\text { Totalmente Insuficiente a } 6=\text { Totalmente Suficiente } \\
1=\text { Totalmente Incomprens. a } 6=\text { Totalmente Comprensible } \\
1=\text { Totalmente Increíble a } 6=\text { Totalmente Creíble } \\
1=\text { Totalmente Inútil a } 6=\text { Totalmente Útil } \\
1=\text { Deficiente a } 6=\text { Sobresaliente }\end{array}$ \\
\hline METODOLOGÍA & $\begin{array}{l}\text { Adecuación } \\
\text { Dinámicas }\end{array}$ & $\begin{array}{l}1=\text { Totalmente Inadecuada a } 6=\text { Totalmente Adecuada } \\
1=\text { Deficiente a } 6=\text { Sobresaliente }\end{array}$ \\
\hline $\begin{array}{l}\text { MANUAL DE } \\
\text { EDUCACIÓN } \\
\text { SANITARIA }\end{array}$ & $\begin{array}{l}\text { Comprensibilidad } \\
\text { Credibilidad } \\
\text { Utilidad } \\
\text { Adecuación }\end{array}$ & $\begin{array}{l}1=\text { Totalmente Incomprens. a } 6=\text { Totalmente Comprensible } \\
1=\text { Totalmente Increíble a } 6=\text { Totalmente Creíble } \\
1=\text { Totalmente Inútil a } 6=\text { Totalmente Útil } \\
1=\text { Totalmente Inadecuado a } 6=\text { Totalmente Adecuado }\end{array}$ \\
\hline VÍDEOS & $\begin{array}{l}\text { Comprensibilidad } \\
\text { Credibilidad } \\
\text { Utilidad } \\
\text { Adecuación }\end{array}$ & $\begin{array}{l}1=\text { Totalmente Incomprens. a } 6=\text { Totalmente Comprensible } \\
1=\text { Totalmente Increíble a } 6=\text { Totalmente Creíble } \\
1=\text { Totalmente Inútil a } 6=\text { Totalmente Útil } \\
1=\text { Totalmente Inadecuado a } 6=\text { Totalmente Adecuado }\end{array}$ \\
\hline APORTACIONES & $\begin{array}{l}\text { Recursos Teóricos } \\
\text { Recursos Técnicos } \\
\text { Diseño de Talleres }\end{array}$ & $\begin{array}{l}1=\text { Deficiente a } 6=\text { Sobresaliente } \\
1=\text { Deficiente a } 6=\text { Sobresaliente } \\
1=\text { Deficiente a } 6=\text { Sobresaliente }\end{array}$ \\
\hline DOCENTES & $\begin{array}{l}\text { Actuación } \\
\text { Claridad } \\
\text { Credibilidad } \\
\text { Empatía }\end{array}$ & $\begin{array}{l}1=\text { Deficiente a } 6=\text { Sobresaliente } \\
1=\text { Nada Claras a } 6=\text { Totalmente Claras } \\
1=\text { Totalmente Increíbles a } 6=\text { Totalmente Creíbles } \\
1=\text { Nada empáticas a } 6=\text { Totalmente empáticas }\end{array}$ \\
\hline SUGERENCIAS & $\begin{array}{l}\text { Aspectos Positivos } \\
\text { Aspectos Negativos }\end{array}$ & $\begin{array}{l}\text { Item abierto } \\
\text { Item abierto }\end{array}$ \\
\hline
\end{tabular}

3. RESULTADOS DE LA EVALUACIÓN DE IMPLEMENTACIÓN DE LOS CURSOS DE FORMACIÓN DE FORMADORES

En este apartado presentamos los datos de la evaluación realizada por los todos los profesionales formados en la $2^{\text {a }}$ fase de este Programa $(n=56)$, que han completado el cuestionario de evaluación al finalizar los Cursos realizados. Al no encontrar diferencias significativas en la valoración de los distintos aspectos del Taller, se presentan los unificadas las respuestas de todos los profesionales, independientemente de en qué semana y en qué orden realizaron los Cursos

\subsection{Aspectos generales del Curso de Formación de Formadores}

El Gráfico 1 recoge la puntuación media general en relación a los Aspectos Generales del Curso (Evaluación Global, Satisfacción, Utilidad y Duración). En general, los profesionales opinan que el haber realizado este Curso ha sido "muy positivo", están "muy satisfechos" y lo consideran "muy útil", pero les ha parecido "corto". 
Gráfico 1: Aspectos Generales del Curso de Formación

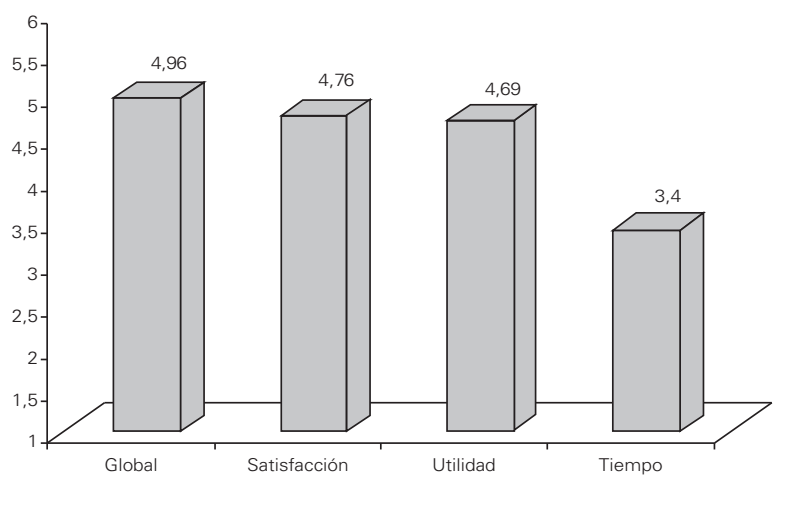

\subsection{Actitud del Grupo durante el Curso de Forma- ción de Formadores}

En el Gráfico 2, se presentan los resultados obtenidos por el Grupo de profesionales en cuanto a la Implicación, Participación e Interés mostrados por ellos durante el Curso. A nivel global, el Grupo percibe que ha sido "participativo" y que se ha mostrado "implicado" y "muy interesado" durante la realización del Curso de Formación de Formadores. Así, podemos decir que estos profesionales han mantenido una actitud positiva y receptiva durante los Cursos.

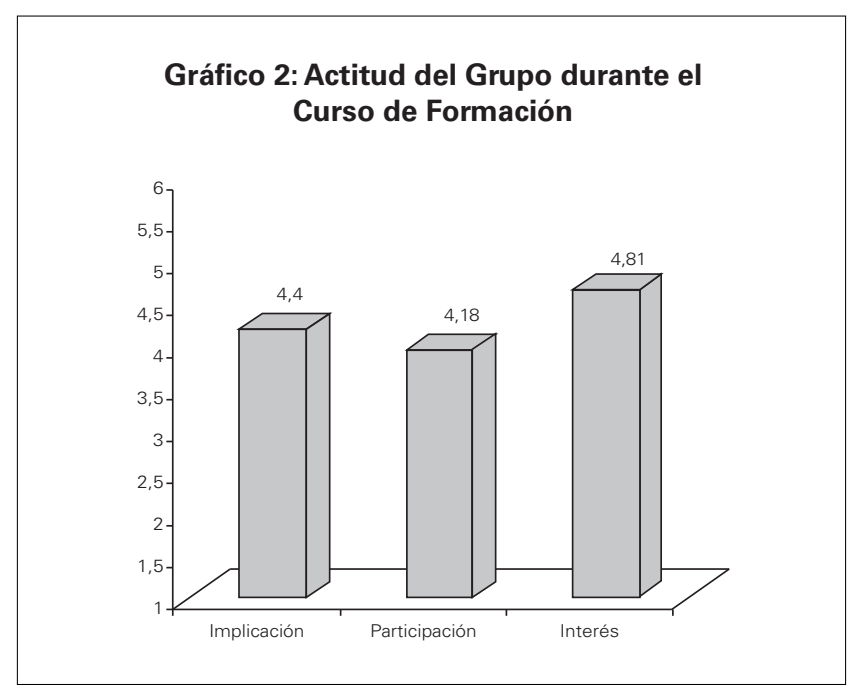

\subsection{Información y Contenidos aportados en el Curso de Formación de Formadores}

El grupo ha valorado la Información proporcionada durante el Curso como "suficiente," "muy comprensible", "muy creíble" y "muy útil."

En cuanto a los contenidos aportados con respecto a cada uno de los diferentes aspectos abordados durante el Curso, los profesionales los evalúan con un "Notable" (ver Gráfico 3).

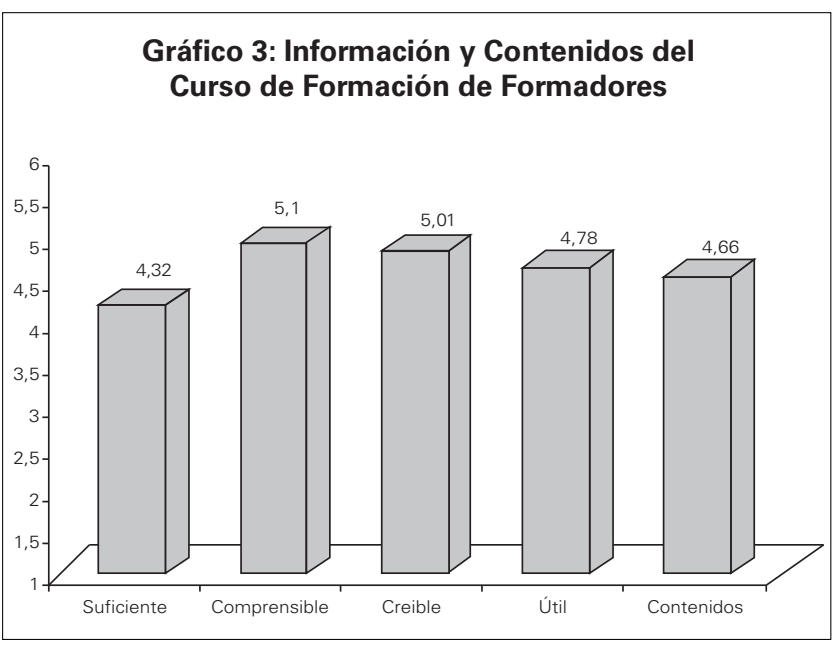

\subsection{Metodología y Dinámicas utilizadas durante el Curso de Formación de Formadores}

En relación a la Metodología y las Dinámicas utilizadas para la implementación del Curso de Formación de Formadores, los profesionales consideran que tanto la Metodología como cada una de las Dinámicas llevadas a cabo durante el Curso "muy adecuadas" (ver Gráfico 4).

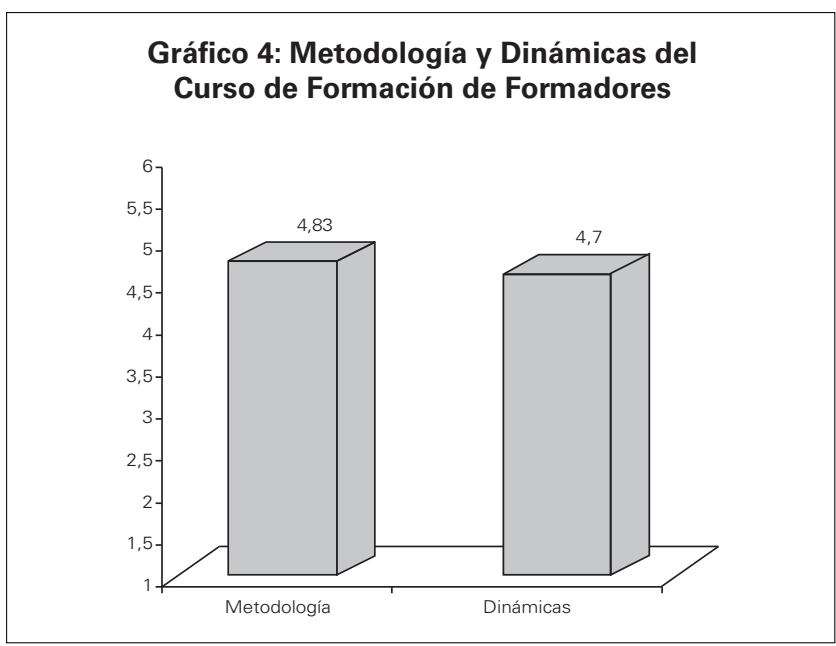

\subsection{Valoración de los Materiales de Apoyo entrega- dos y utilizados durante el Curso de Forma- ción de Formadores}

En los Gráficos 5 y 6, se presenta la valoración de los materiales de apoyo (vídeos "Hacia un Sexo Más Seguro" y "Si te metes, no te mates" y Manual de Educación Sanitaria) hecha por el Grupo de profesionales en cuanto a la Comprensibilidad, Credibilidad, Utilidad y Adecuación. 
Con respecto a los vídeos, a nivel general, el Grupo percibe que son "muy comprensibles", "muy creíbles", "muy útiles" y "muy adecuados" (ver Gráfico 5).

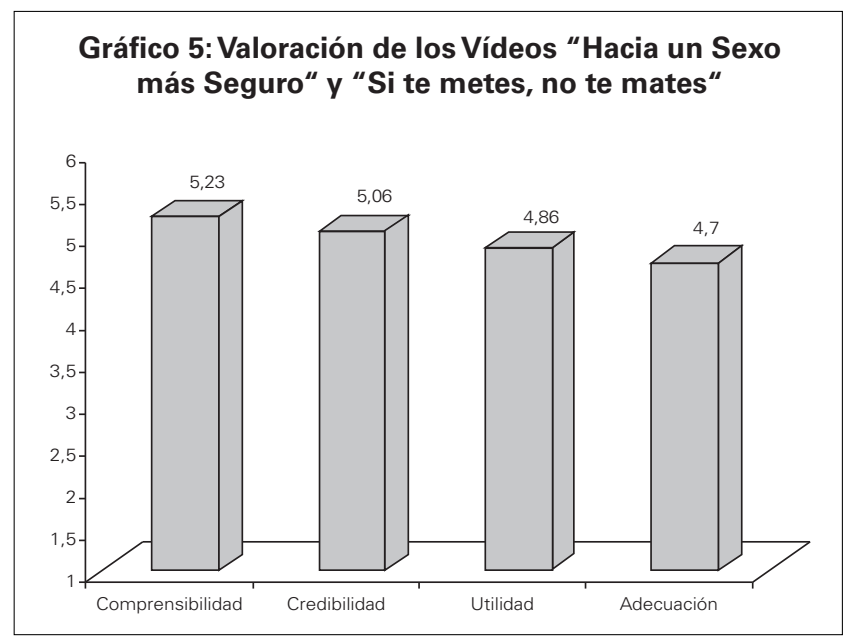

El Manual de Educación Sanitaria (Insúa, 1999a), consta de tres partes. La primera parte trata los aspectos teóricos y prácticos que son comunes a los TSMS y a los TCMR (como la normativa, difusión y duración de los talleres; características de los usuarios de drogas que van a participar en ellos y las características que debe reunir el formador en un Taller). La segunda parte es específica de los TSMS, y trata temas relacionados con los objetivos de estos talleres, con las conductas sexuales de riesgo de los usuarios de drogas, con los contenidos que deben ser abordados, y en qué línea, en los TSMS. La tercera parte, es específica de los TCMR y trata acerca de los objetivos que se persiguen en estos talleres, de los daños a la salud asociados al consumo endovenoso de drogas, de los determinantes de los comportamientos de consumo y de los contenidos que debe tener un Taller de consumo de menor riesgo.

Las tres partes del Manual han sido valoradas con un notable. Como se observa en el gráfico 6, los profesionales formados lo consideran muy comprensible, muy creíble, muy útil y muy adecuado (ver Gráfico 6).

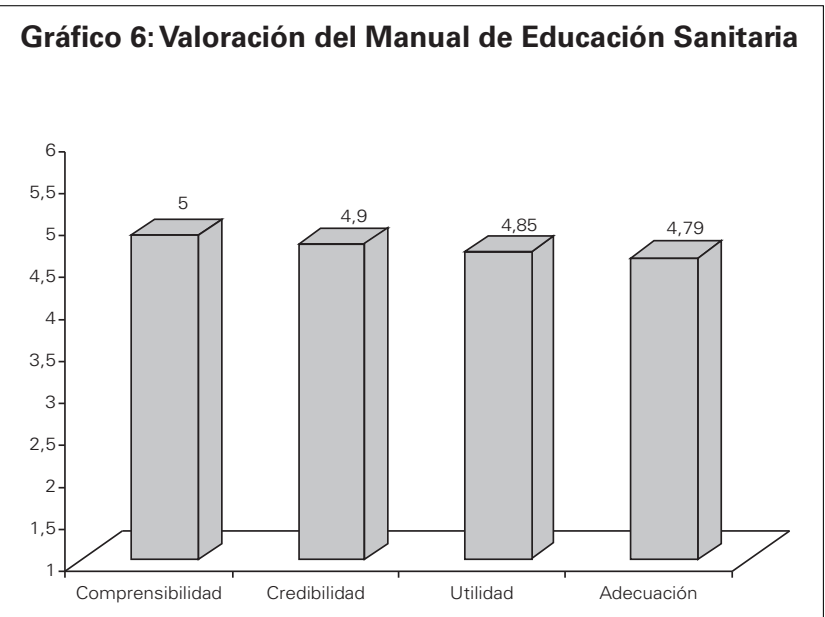

\subsection{Percepción de las Habilidades aportadas por el Curso de Formación de Formadores}

Una vez realizado el Curso, el Grupo califica sus conocimientos, recursos técnicos y su capacidad de diseñar e implementar tanto Talleres de Sexo más Seguro como Talleres de Consumo de menor Riesgo con una nota media que se sitúa entre "Bien y Notable" (ver Gráfico 7).

\section{Gráfico 7: Habilidades Percibidas para la Realización} de TSMS y TCMR

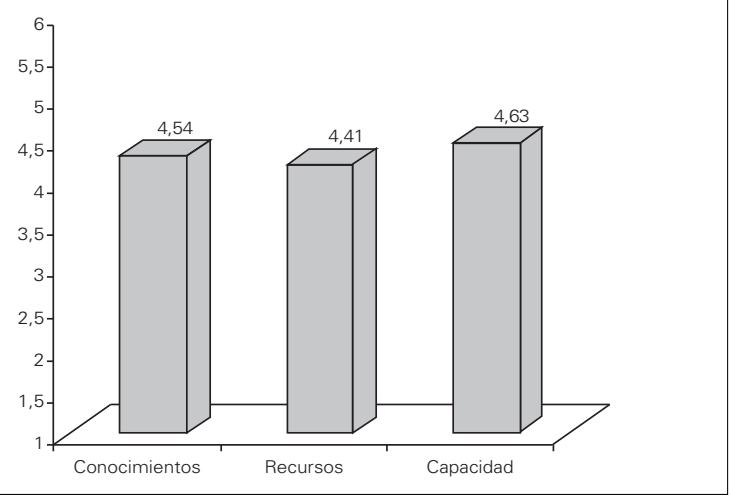

\subsection{Valoración de los Docentes encargados de impartir el Curso de Formación de Formadores}

En el Gráfico 8 se presentan las puntuaciones dadas a los docentes en cuanto a su Actuación, Claridad, Credibilidad y Empatía. A nivel general, el Grupo percibe que éstos han sido "muy claros", "muy creíbles" y "muy empáticos", y califican su actuación con un "Notable".

Gráfico 8: Valoración de los Docentes encargados del Curso de Formación

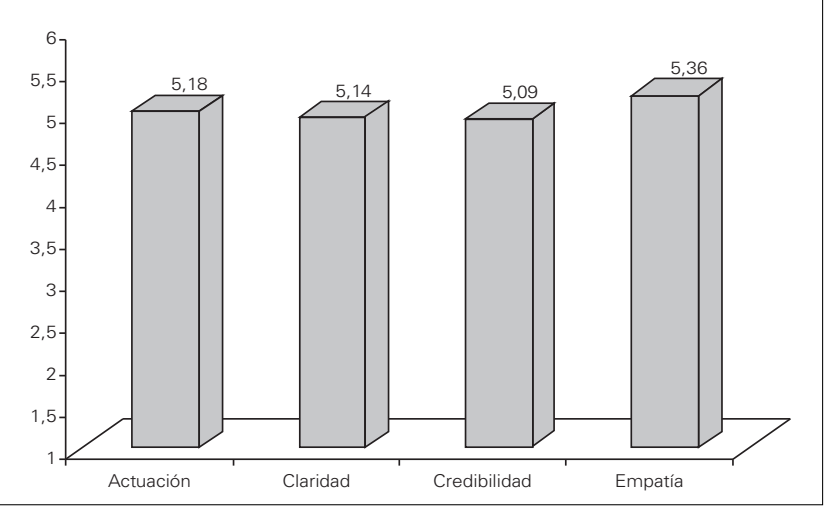

\section{DISCUSIÓNY CONCLUSIONES}

La evaluación de implementación del Curso de Formación de Formadores diseñado para profesionales 
sanitarios que trabajan con el colectivo de usuarios de drogas, evidencia que todos los profesionales formados realizan una valoración positiva de las características estructurales del mismo.

Con respecto a la evaluación cualitativa sobre los aspectos positivos y negativos del Curso, un 30\% de los profesionales valora especialmente la buena estructuración y metodología del mismo que ha permitido proporcionar una gran cantidad de contenidos y recursos muy útiles para la consecución de los objetivos de esta Formación. Asimismo, se menciona como positivo la presentación de diferentes dinámicas grupales a través de un método predominantemente participativo y ameno y el ambiente cohesionado de los grupos que ha permitido un buen aprovechamiento del Curso.

Asimismo, un 34\% de los profesionales considera que el tiempo dedicado a esta Formación ha sido escaso y un $21 \%$ demanda más entrenamiento para profundizar en dinámicas dirigidas al cambio de actitudes y conductas de riesgo, evaluación de programas, contingencias que aparecen en el trabajo con grupos de usuarios de drogas, diseño de talleres y habilidades para impartir este tipo de programas de prevención. Es una constante en este tipo de Talleres la demanda de mayor tiempo de entrenamiento ya que las mayores dudas aparecen siempre sobre la propia capacidad para ser formador. No obstante, los asistentes consideran que los docentes les han motivado suficientemente para seguir ampliando conocimientos sobre el tema a través del Manual de Educación Sanitaria de cara a diseñar, implementar y evaluar Programas de Prevención de los problemas de salud asociados al uso de drogas dentro del marco teórico de la reducción del daño.

Estos datos nos permiten sostener que el Curso de Formación de Formadores ha sido bien diseñado y que su estructura permite trabajar en profundidad distintos aspectos que van más allá de la información, y que van a tener un papel fundamental a la hora de enseñar cómo poner en marcha Programas de Prevención.

Con respecto a la viabilidad de la estrategia de redes, estos 56 profesionales sanitarios, después de la supervisión y el diseño de sus propios Talleres, han replicado en sus respectivas CC.AA. estos Programas de Formación, encontrándonos con que tras un año de trabajo, 684 profesionales que trabajan con usuarios de drogas en todo el Estado, han adquirido la formación necesaria para poner en marcha Programas de prevención de los problemas de salud asociados al uso de drogas. Es decir, se ha multiplicado por 12 la población formada a través de la estrategia de redes, lo que nos confirma la idoneidad de este tipo de trabajo, que es altamente rentable cuando está bien coordinado y organizado.

Creemos que ahora el desafío pasa por implementar sistemáticamente este tipo de programas en los centros de atención a drogodependientes; es decir, por incorporarlos como una de las ofertas sanitarias básicas para el usuario, al igual que los Programas libres de drogas, los programas de sustitutivos opiáceos u otro tipo de Programas de Educación Sanitaria; ya que solamente cambiando los comportamientos de riesgo (tanto los sexuales como los de inyección) conseguiremos reducir los daños a la salud asociados al uso de drogas en el colectivo de usuarios de nuestro país.

\section{BIBLIOGRAFÍA}

Bayés, R. (1995). Sida y Psicología. Barcelona, Martinez Roca.

Coyle, S.L.; Boruch, R.F. y Turner, C.F. (Eds.). (1991). Evaluating Aids Prevention Programs. Washington DC, National Academy Press.

De Andrés, M. (1999). Si te metes, no te mates. Vídeo educativo. Barcelona, Ex Aequo

Des Jairlais, D.C.; Friedman, S.; Perlis, T. et al, (1999). Behavior and HIV infection among new drug injectors in the era of AIDS in New York City. Journal of Acquir. Immune Defic. Syndrome Hum. Retrovirology, 20, 67-72.

Freudenberg, N. (1990). Developing a new agenda for the evaluation of AIDS education. Health Education Research, 5, 295-298.

Insúa, P. (1999a). Manual de Educación Sanitaria: Recursos para diseñar Talleres de prevención con usuarios de drogas. Madrid, Plan Nacional sobre Drogas.

Insúa, P. (1999b). Procesos cognitivos y conducta: su trabajo en los programas de formación de formadores. Investigació Psicológica, 71, 54-59.

Insúa, P. (1999c). Hacia un sexo más seguro. Vídeo psicoeducativo. Madrid, Plan Nacional sobre Drogas.

Insúa, P. y Moncada, S. (en prensa). Está cambiando el discurso sobre el uso de drogas en las Instituciones? Un Programa de Formación de Formadores. Revista Española de Drogodependencias.

Mantell, J.; DiVittis, A. y Auerbach, M. (1997) Evaluating HIV Prevention Interventions. New York, Plenum Press.

Miller, R.L. y Cassel, B.J. (en prensa). Ongoing evaluation in AIDS service organizations: Building meaningfull evaluation activities. Journal of Prevention in the Community.

Rossi, P.H. (1993). Evaluation: A systematic approach. Newbury Park, CA: Sage

US Conference of Mayors (1990, Diciembre). Evaluation for HIV/AIDS Prevention Programs. Technical Assistance Reports. Pp.1-12. 